\title{
Validity Of Scoring Methods In The Presence Of Outliers
}

\author{
Nicole Uhde, Westfälische Wilhelms-Universität Münster, Germany
}

\begin{abstract}
Rankings enjoy growing popularity in the economical sciences. Well known institutions like the World Economic Forum, Heritage Foundation and the OECD make use of rankings to exert competitive pressure on the ranked countries. To achieve any such desired effects rankings need to be accepted and approved as a whole, and in particular regarding the applied methodology. In order to appeal to wide sections of the population scoring methods are applied to aggregate a composite indicator. Experience has shown that outliers have a distorting effect on the ranking order and therefore cause economically implausible results which are a target for criticism. For these reasons the choice of an adequate scoring method is of great importance. The applied technique should provide a feature which enables it to mitigate the distorting effect of outliers without the necessity for an arbitrarily elimination of data points. Although scoring methods have a high influence on ranking results, scientific analysis is often more concerned with the optimal choice of indicators or the weighting scheme, whereas the impact of extreme values is not addressed. According to this, the present research is related to the question which scoring method is the best choice in the presence of outliers. Evidence is given, that Logistic Function Methods have the ability to mitigate outlier distortion effects. The analysis approaches the issue considering two aspects: It combines the theoretically derivation of scoring methods' statistic strengths and weaknesses for the ranking process and highlights the bootstrap technique to assess the validity of score results in the presence of outliers.
\end{abstract}

\section{INTRODUCTION}

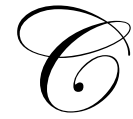

onstructing composite indicators by the use of aggregated score rankings is a central feature of many rankings. For a long time the World Economic Forum has been publishing the Global Competitiveness Index which measures the competitiveness of 125 countries (in 2006). Recently the European Union $(E U)$ has also started using rankings in their working area. In Germany, the Bertelsmann Foundation has published its own country study - the Bertelsmann Location Ranking (Bertelsmann 2007). A great of well-known rankings utilize scores, which are obtained by transforming measurement values. Presenting the results by way of score values is a means of presenting complex economic contents in a simplified form to a general public without economic education but with an interest in country rankings. Consequentially, rankings may help to reinforce discussions about the strengths and weaknesses of governments and exert a certain competitive pressure on them. Although scoring methods are of a not unsubstantial importance, scientific analysis is more concerned with the optimal choice of indicators, the weighting scheme or the data collection process (Van Suntum 2004; Kladroba 2005 and 2005a; Bayer 2000). Matthes and Schröder (2004) have, for the first time compared effects of different scoring methods on an aggregated ranking.

With the help of numeric examples they show that the position of single countries can change more or less depending on the employed scoring method. This provides critics with a target and undermines the potential need for action of those responsible. The choice of a non-arbitrary scoring method is of major importance, regarding these considerations. The existence of outliers needs careful handling of the data set and even more a wise decision as to the method to be employed. 
For the first time scoring methods will be presented and tested according to their ability to mitigate outlier distortion effects. In a descriptive approach strengths and weaknesses of all techniques are explained and referred to special statistic characteristics. The results will be proofed formally by the use of bootstrap confidence intervals which gives evidence that scoring methods exist which work well in the presence of outlier-biased data sets.

\section{BASIC DEMANDS CONCERNING STRUCTURE AND METHODOLOGY OF RANKINGS}

The aggregation of a final rank order using a set of individual indicators (indexmethod) is applied in many well-known country rankings, which quantify economic success or competitiveness of countries. These multiindicator target dimensions can not be observed and measured in reality and so have to be addressed by the use of several single indicators which contribute to explain the target dimension.

Van Suntum (2004: 4) frames the condition that the target dimension has to be clear and conclusive and should be well distinguishable from other targets. The choice of single indicators requires economic plausibility and has to be based on economic theory and empirical analysis. As well, the weighting scheme should not be random but selected with the help of an econometric study. Furthermore, to reproduce the results it is important that the original data can be recalculated from the aggregated ranking.

Aggregating single indicators offers a wide scope for arbitrary weightings and allocation of scores which has a strong influence on the results of the ranking. To build a less vulnerable ranking methodology, we need to provide stringent reasons for the application of a certain scoring method. Aware, that structure and variance of the measured data have an impact on the optimal choice of a scoring method and in addition to that, every scoring method is alterable concerning the determination of its parameters, it is simply impossible to find an absolutely nonarbitrary scoring method which is the best choice for all kinds ${ }^{1}$ of indicators. Many decisions being made in the process of choosing a scoring method are subject to the individual preferences of those persons who do the ranking. We have to decide for example, if the large bandwidth of a measured data set is a result of the existence of outliers or if it just mirrors a "normal" data spectrum. In the last case extremely high (or low) values would be considered as preferable benchmarks. Following Arrow (1963) it is impossible to find the perfect solution for the preference decision process.

According to the Arrow-Theorem, it is much more important to justify the choice and implementation of a certain scoring method with its statistical features. The following minimum requirements for scoring methods can be derived:

- $\quad$ Independence from the dimension of the data set

- Depiction of economic differences of data points in the score results

- $\quad$ Application of qualitative data

- $\quad$ Selection must depend on statistical features which fit to the data set's structure

Different dimensions in the underlying data structure have to be standardized using an appropriate method to make indicator results comparable. Linear transformations are frequently applied and proposed in $O E C D$ 's Handbook on Constructing Composite Indicators (Giovannini et. Al. 2005: 18). Knowing that certain standardization techniques have an impact on the final ranking order Giovannini et Al. recommend the implication of a method which will transform the measured data on a scale with zero mean and standard deviation of one.

Scoring results should reflect the economic differences between data points. This is important for data sets which are more or less evenly distributed and even more compelling for those series of measurements which contain extreme values and are asymmetrically distributed (Matthes / Schröder 2004: 3). Expressing the economic picture in the ranking is a major requirement to scoring methods and crucial for validity and reliability of a ranking. Eventually the capability of a scoring method determines the acceptance of the ranking. If the measured values are biased by

1 Independent from number and characteristic of single indicators, bandwidth of the data set, flow or stock values time series or cross-section data. 
outliers, "normal" data points (which lie in the expected bandwidth) seem to be disproportionally high or low compared to the extreme values (see section 3). A proper scoring technique should control for the distorting effect of outliers in a way, that point scores will not reflect the bias of the normal data.

Another preferable characteristic of scoring methods is related to the problem of qualitative and quantitative data. Qualitative survey data are typically not numeric and need special treatment to transform it into point scores. For reasons of transparency and consistency it is desirable to find a way to employ the chosen scoring method on survey data as well. An exemplary approach of a scoring process with qualitative data is given in section 3.4.2.

Transforming measured values into point scores can be achieved by several scoring methods which differ in their functional form and therefore in their statistical features. Some techniques are accompanied by a leveling of relative distances in the data or they suffer from a strong arbitrariness because parameters have to be set manually. Those statistical characteristics can be derived from the functional form of the method and have to be considered in the ranking process. The attributes of different scoring methods are outlined in the next section.

\section{CURRENT SCORING METHODS}

\subsection{Rank Sum Method}

The Rank Sum Method (RSM) requires a sorted list of data points. The best result has the highest rank and receives a point value according to the number of results in the data set. Assuming a ranking indicator with twenty elements the best result would gain 20 points and the worst result one point. After the scoring procedure all objects have the same score difference of one (point) which implies that the original data scaling might change from a metric or interval to a more simple ordinal scale. For example a sample of rates of inflation has an interval scale whereas the final rank order is ordinally scaled. The lower scale causes a loss of information on the relative distances of the original values. Distances between the individual score values can not be interpreted adequately. Furthermore, neglecting the relative distances gives a wrong impression of the success and weaknesses of the ranked objects. This is a special problem of multi-indicator rankings where single indicators are aggregated to a composite indicator. The accumulation of omitted information may have a strong influence on the final ranking order.

Matthes and Schröder (2004) demonstrate that the choice of a scoring method is critical for the overall ranking. They present a simple example of two indicators which are aggregated to a final ranking order. The indicators are constructed in such a way that the result is one single "true" ranking order. The Rank Sum Method fails to produce the result due to the omission of relative distances between data points.

\subsection{Scoring Class Method}

Scoring methods which allocate scores according to formerly built classes are called Scoring Class Methods (SCM). All measured data which correspond to a special class are provided with the same score value independent from the relative distances. Alternatively the method can be adjusted to ensure that there is no information loss. In this case each class contains a span of scores which are allocated to the data points according to their relative distances. For example countries with a rate of inflation between $0 \%$ and $2 \%$ will fall into the first which allocates $100-91$ points. However there are breaks in the point allocation at the crossover point of two classes. A country with a measured rate of inflation of $2.01 \%$ for example will be located to the second class with scores ranging from $90-81$. Some of the indicators applied by the Heritage Foundation's in their Index of Economic Freedom (Kane et al. 2007) were based on a Scoring Class Method until the year 2007 when a mix of linear and non-linear scoring methods as well as Scoring Class methods was introduced. Qualitative indicators which are often devised on the basis of interviews and opinion surveys can be applied with the SCM. For this purpose, purely verbal answers are sorted into previously constructed classes of theoretical answers. Each of the invented classes has an attached value 
which has the function of transforming answers into score values. ${ }^{2}$ The definition of classes causes problems because score leaps will be present at the classes' cross-over points. A loss of relative distances in the original data due to the crossover between the classes may have to be anticipated, and hence a possible loss of information.

As a result, it will be impossible to infer on the original data from the score values. One possible solution may lie in the fact that classes can be constructed as narrow as the ranker decides to control for the information loss but still the disadvantage remains that class boundaries and associated scores have to be developed manually. Ultimately, it is the ranker who decides on the optimal number and length of classes and if classes possess fixed score values or a score value range. Similar to the RSM interval scales of the original data might be down-scaled to simple ordinal scales. ${ }^{3}$

\subsection{Linear Scoring Methods}

Using metric techniques helps to take relative distances into account which is a valuable statistical feature of these scoring methods. Measured data are interpolated linearly and transformed to a freely chosen scale. Interpolation techniques in practice are often available in the following functional form:

$$
\mathrm{x}=\frac{a-x_{i}}{b}
$$

with $\mathrm{x}=$ transformed value, $\mathrm{x}_{i}=$ particular $\mathrm{x}$-value from 1 to $\mathrm{i}$, $\mathrm{a}$ and $\mathrm{b}=$ constant. Given that $\mathrm{a}$ is the minimum (maximum) value and $\mathrm{b}$ is the span between minimum and maximum can be expressed formally as:

$$
\mathrm{x}=\frac{x_{i}-x_{\min }}{x_{\max }-x_{\min }}
$$

for indicators where high values mean positive results and

$$
\mathrm{x}=\frac{x_{i}-x_{\max }}{x_{\max }-x_{\min }}
$$

if low values are preferred.

Each value $x_{i}$ is set in proportion to the span of the marginal values (so called "min-max method" by Ochel / Röhn 2006: 52). In a second variant the mean of the data set has been inserted for the parameter a and the standard deviation for $b$. The resulting scale of standardized values has a mean of zero and a standard deviation of one:

$$
\mathrm{x}=\frac{\bar{x}-x_{i}}{\sigma_{x}} .
$$

The benefit of this approach is that all single indicators have the same mean and standard deviation which improves comparability and facilitates the subsequent aggregation of a composite indicator. Furthermore, standardization techniques do not alter the data scaling and do not cause information losses.

Some problems are related to linear (or metric) scoring methods of which one is that metric techniques are extremely susceptible to outliers. The value of $b$ in (1) increases as an effect of the increasing span between (outlier

2 The approach is applied by the Heritage Foundation to allocate scores to the indicator of "property rights" which is calculated to assess the embodiment of property rights.

3 Except from nominal scaled qualitative data. 
biased) minimum and maximum value and of the growing variance. Furthermore, outliers have distortion effects on those values that lie closely together. Especially data points in the midfield of a data set are compressed by the large weight of extreme values. Small, but significant differences of indicator values get lost so that subtle content-related differences can not be distinguished by their score value any more. Extreme values receive the maximum or minimum score value and show large score differences towards the next normal values which might, however, be very good or bad results.

\subsection{The Logistic Function Method}

\subsubsection{Shape And Curvature}

Logistic functions are so called sigmoid functions featuring an s-shaped curve which is realized by the division of the numerator by an exponent. The function has been applied in Natural Sciences for a long time and was used to depict bacterial growth processes in its first applications. ${ }^{4}$ In it's mathematically simplest form the logistic function is given as:

$$
\mathrm{P}(\mathrm{z})=\frac{1}{1+e^{-z}},
$$

where $\mathrm{z}$ is an input variable which produces output $\mathrm{P}$. Transferred to the field of rankings standardized values are inserted to $\mathrm{z}$ and the output can be interpreted as resulting score value. The data points are transformed sigmoidally and build the known s-shaped curve. The Logistic Function Method has the characteristic to transform indicator values in a way that they can be compared against each other regardless of their original dimension, bandwidth and variance. The ranking is not influenced by the s-shaped data transformation even so the absolute and relative differences of data are affected. It is possible to choose any scale of positive score values. A common scale has proofed beneficial for the aggregation of composite indicators.

Before indicator values can be inserted into the function the need to be standardized. As described in section 3.3 the data points are set in proportion to the mean and standard deviation to obtain zero mean and a standard deviation of one. As described above the standardization method has weak results if an indicator has a greater number of data points located around the mean and some outliers which affect an increasing bandwidth. In this case the nominator value increases while the majority of values $x_{i}$ are small. Again, the interpolation result consists of many small values which tend to be compressed. The distortion effect of outliers can not be wholly mitigated by the use of interpolation techniques but at least in parts by the application of a logistic function which allocates an overproportional amount of scores to the values in the midfield. As a result, even if values are compressed they can be distinguished by their score value. Matthes and Schröder (2004) prove that a logistic scoring method contributes to the compensation of outliers due to its s-shaped run of the curve. The point allocation depends on the function's slope in a way that the compressed $\mathrm{z}$-values are located on the steep part of the logistic curve where marginal changes in the $\mathrm{z}$-value effect large differences in the score value due to the prevailing high slope. On the other side high standardized values will be located on the asymptotical parts of the curve and receive relatively sparse point growth rates for a given marginal change. Accordingly, the transition from very good (bad) to even exceeding results will not be benefitted (penalized) by a high score difference. Very good (bad) results claim almost maximum (minimum) score values and scores of midfield results get uncompressed which makes good economic sense.

Different variants can be deviated from the basic logistic function given in (5). All functions utilize one ore more parameters which constitute location and curvature of the graph. The basic function and two modifications are presented in figure 1.

4 One bacterium generates a small bacterial culture at first, but due to their exponential growth the culture becomes rapidly larger. After some time growth rates decrease as a matter of arising concurrence between the single bacteria. At least a natural saturation level is reached. 
The light grey line is the graph of the basic logistic function with inflection point at $(0 ; 0.5)$. The black line presents a modified run of the curve after having added a number $>0$ to the term in (5). Multiplication by a number $>0$ causes a faster asymptotical convergence which is accompanied by a higher slope in the midfield of the curve. Adding and adjusting more parameters will result in a variety of new functions which will serve different purposes. Score results can, for example, be located to so called traffic light segments which send ranking addressees a signal of need for action. Accordingly, the red segment draws attention to below-average results whereas the green segment speaks in favor of the economic success of the ranked institutions or countries. It can be generally stated that given a high compression effect of outliers, parameters should be specified in a way that the curve converges rapidly to the asymptotes.

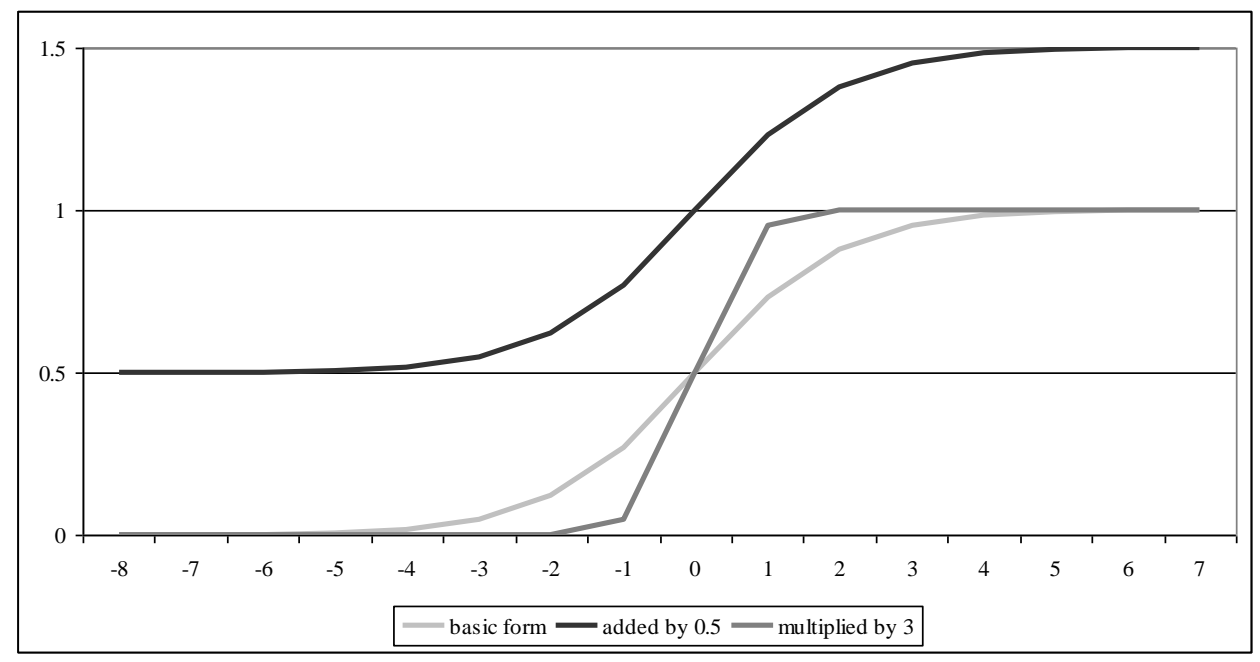

Figure 1: Parameter impact on location and curvature of the logistic function.

\subsubsection{Utilisation Of Qualitative Data}

The utilization of qualitative data using the logistic scoring method is possible but not free of problems. The possibility of maintaining a consistent method of valuation will be demonstrated on the basis of an example. For an economic ranking it could be interesting to learn something about the assessment of a group of experts on the future development of a country regarding educational topics and availability of qualified employees. The following three answers could be anticipated:

1) In the nearest future the country will have to report a slight decline in qualified employees, which will dissolve after a short period of adjustment within the sector of education. This is because it will be easy to find students to overcome the shortage without - at the same time - creating a shortage of qualified employees in a different sector.

2) In the nearest future the country will have to report a strong decline in qualified employees, which will not even dissolve completely, if more students enrol in the relevant subjects, because the migration of students from other subjects will provoke a shortage in those areas.

3) The country will have to report an enormous shortage in qualified employees and the system of education is furthermore extremely inflexible and does not contribute to solve the problem.

All answers are very distinct and shall be transformed in a scoring method, which has been designed for numeric measured data. A solution could be to create a broad pattern first, which contains every possible combination of answers. The actual given answers should then be allocated to the scheme of possible answers. Every answer will be assigned with a value, which expresses how close the answer comes to the theoretically best possible answer. The theoretical answer "The system of education carries on without a lack of intellectual quality / education and an 
appearing lack could easily be solved by migration of human capital into the relevant sectors" would be in the vanguard of the pattern and is assigned with the highest value. In case of ten classes, this would be a value of ten, for example. The next gradation within the possibilities of answers would receive a value of 9 , and so on. All survey results are sorted into the classes.

Because every method has been ascribed a value in the end, a numeric scoring method can be applied in the next step. Given that three answers have reached values of 9,2 and 1, then these three values would be converted into point values using the Logistic Function Method. It is also possible to use the logistic method with non-numeric indicators, as long as these are measured on ordinal scales, thus permitting a logic order like "well, bad, worst". It must however be objected, that the introduced method is not free of arbitrariness, because creating scoring classes and assigning the answers to the classes is will be influenced by the subjectivity of the executing person. To avoid criticism in this direction an independent party is entrusted to create the scoring classes and assign actual answers to the classes.

\section{THE CHOICE OF A SCORING METHOD IN THE PRESENCE OF OUTLIER-BIASED DATA SETS}

\subsection{Samples With Outlier Distortion Effects}

A common problem with the data set (sample) of a ranking is a distortion effect initiated by outliers. ${ }^{5}$ Barnett and Lewis (1978) were the first to mention the necessity of a careful data analysis for the detection of outliers using appropriate techniques. Otherwise, they concluded, outliers would systematically distort the research results. The same applies to statistics like the mean or confidence intervals which present classic inference methods.

Generally, outliers are those "observations which appear to be inconsistent with the remainder of that set of data" (Barnett / Lewis 1978: 4 and alike Grubbs 1969). A more precise definition by Belsley (1980) says that outliers are "subsets of the data that appear to have a disproportionate influence on the estimated model". Furthermore, we need to distinguish between true and false outliers. Some extreme values are a result of measurement errors, transmittal errors or the false choice of a statistic model. Values might be calculated incorrectly or they were faultily extracted from the data source. On the other side, an apparent outlier might be characterized as a normal value if another distribution for the sample is taken as a basis. The last issue is extremely important because observations might be mistakenly declared as outliers, due to a misleadingly specified distribution of the data set. A "false outlier" is therefore defined as a data point which is part of a certain (unknown) population but was detected as a "true" outlier as a result of either a wrong assumption of the sample's underlying distribution or an improper detection method. For example if we assume the sample to be normally distributed then extreme (marginal) values will be classified as outliers more probably than on the assumption of a Cauchy-distribution (Buttler 1996: 9).

Far too often, the elimination of outliers is postulated in literature and used in rankings. This procedure requires a sensitive and correct determination of the underlying distribution of the data set. Only then the right test for outlier detection can be selected and elimination of the discovered values is tolerable. Different tests for the detection of outliers can lead to different results because they rest upon varying distributional assumptions. In the worst case, using a wrong test may lead to normal values being classified incorrectly as outliers and eliminated in the data set. Determination of the distribution and choice of a proper test are complex procedures and offer ample room for errors. Especially in the case of small samples (around 20 countries) the identification of the underlying distribution is a difficult task. For this reason it is advisable to abandon elimination techniques as far as possible. Barnett and Lewis (1978: 4) already postulated that highly diverging values should not be eliminated in the data analysis and propose various methods of handling outliers. Looking at multivariate regression models it becomes apparent that detection and handling of outliers is broadly discussed in literature (Barnett / Lewis 1978; Martin 2005; Sturm / Hahn 2000 and others). Most rankings refer to univariate data analysis while reciprocal influences of the individual indicators (measured by the correlation) pass unheeded. Analyzing current literature offers no advice for the han-

5 In the following the term ,outliers“ will be defined as those values which stay in significant contrast to the rest of the data set. A differentiation between marginal values and outliers as in Barnett / Lewis (1994) is not applied because typically outliers and extreme marginal values are the same. 
dling of outliers in rankings. Moreover many rankings are confined to the mere elimination of striking values without executing an outlier detection test.

In the $O E C D$ 's Handbook on Constructing Composite Indicators (2005: 18) the proposal is put forward to eliminate extreme values if they are not an explicit requirement for benchmarking reasons. An arbitrary elimination or manipulation of data points has to be assessed critically. Any interference constitutes a breach with the principle of non-arbitrariness and the ranking would incur censure. If the ranking order (and point allocation) has to satisfy requirements of validity and reliability than it is most important to make use of a method which offers the ability to compensate for outliers in order to abstain from a random data elimination. The existence of that kind of technique is demonstrated in the following sections.

In the next section an example of a real outlier biased data set is given and tested as to which scoring method produces the most valid results, when applied to the sample.

\subsection{The Validity Of Score Values Referred To The Sample Of Per Capita Debt}

The four scoring methods which were introduced in section 3 are brought up to assess their validity as described above. As the result of its statistical features, mentioned in 3.4.1 and 4.2 the Logistic Function Method seems to be an auspicious approach. The Linear Function Method is a representative technique of many rankings because it is easy to handle. Finally, the Rank Sum Method and Scoring Class Method are applied on the data set. To examine the Logistic Function Method, two functions with different parameters were chosen. The first one (LF1) is the following function which was introduced by Matthes and Schröder (2004: $12 \mathrm{ff}$.):

where $\mathrm{x}_{i}=$ measured values, $\tilde{x}=$ median and

$$
\mathrm{F}(\mathrm{z})=\frac{1}{1+e^{-c z}}
$$

$\mathrm{MAD}=$ mean distance of measured values to median according to amount.

The transformation of indicator values into the intermediate variable $\mathrm{z}$ causes the measured values to be centered on a standardized scale around the median. The advantage of an alignment around the median (instead of mean) is that as many indicator values as possible come close to zero, even if the data set is skewed. ${ }^{7}$

The second logistic function was introduced by van Suntum (2004) and is used by the Bertelsmann Stiftung in their business location ranking "Internationales Standort Ranking". The purpose-built Logistic Function Method serves to assign indicator values to a so called traffic light scheme. According to this, above-average data points ${ }^{8}$ are allocated to a green segment, average values are located into a yellow segment and poor results are allocated to the red traffic light segment which implies urgent need for action. The allocation and centering of scores is achieved by adjusting the parameters $\mathrm{a}-\mathrm{d}$. The determination of parameter values is conducted non-arbitrarily by the use of a software based iteration process. ${ }^{9}$ The Logistic Function Method (LF2) reads as follows:

6 The prefactor of -1 effects large positive data points (a high value of per capita debt) to be allocated with a small score value. If large values are treated as preferable results, the multiplication is not applicable.

7 The z-value of the median amounts to zero. Marginal deviations from the median maintain explanatory power.

8 These are such values, which are less than one standard deviation away from the mean of the three top countries.

9 For further information see Bertelsmann Stiftung (2007). 


$$
\mathrm{F}(\mathrm{z})=a * \frac{e^{b^{*} z}-c}{e^{b^{*} z}+d}+a * \frac{c}{d}
$$

Once the parameters are determined it is possible to apply the functional form on all individual indicators and all times which ensures largest possible comparability.

Rankings on the competitiveness of countries or federal states are often based on small samples which build the ranking order. An example of a small and outlier biased sample is the following indicator of per capita debt in Germany's federal states: ${ }^{10}$

$$
\mathrm{x}=\left(\mathrm{x}_{1} ; \mathrm{x}_{2} ; \ldots ; \mathrm{x}_{16}\right),
$$

with $\mathrm{x}=(1,851 ; 2,828 ; 3,683 ; 4,963 ; 5,986 ; 6,033 ; 6,070 ; 6,313 ; 6,446 ; 6,615 ; 7,384 ; 7,716$;

$$
7,783 ; 12,134 ; 16,901 ; 18,557) \text {. }
$$

Evidence suggests that the presented sample might be used as ranking indicator. To give an example of how scoring methods work in reality the techniques will be applied on the data set later. A successful scoring method will produce a point allocation which functions to depict a realistic and valid overall picture. Therefore, scoring methods need to have the following characteristics:

- $\quad$ The method has to be non-arbitrary and comprehensible

- $\quad$ Outliers must not be eliminated or modified manually

- $\quad$ Outlier scores should not differ too much from the score value of the next normal data point

- Score compression within the data points around the median should be avoided

- $\quad$ Score values need to be economically plausible.

The next table presents score values of the per capita debt sample. The results are assessed by the requested characteristics which were just developed.

\begin{tabular}{|c|c|c|c|c|c|c|}
\hline \multicolumn{7}{|c|}{ Score values } \\
\hline Federal states & Per capita debt in 1.000 $\mathbf{~ L F 1 ~}$ & LF2 & Linear function & RSM & SCM \\
\hline 1 & 1,851 & 99.96 & 100.00 & 100.00 & 100 & 100 \\
2 & 2,828 & 99.76 & 100.00 & 94.15 & 93.33 & 90 \\
3 & 3,683 & 99.00 & 99.99 & 89.03 & 86.67 & 80 \\
4 & 4,963 & 91.77 & 99.46 & 81.37 & 80.00 & 70 \\
5 & 5,986 & 66.15 & 85.26 & 75.25 & 73.33 & 60 \\
6 & 6,033 & 64.33 & 83.15 & 74.97 & 66.67 & 50 \\
7 & 6,070 & 62.88 & 81.32 & 74.75 & 60.00 & 50 \\
8 & 6,313 & 52.83 & 65.68 & 73.29 & 53.33 & 50 \\
9 & 6,446 & 47.17 & 54.96 & 72.49 & 46.67 & 50 \\
10 & 6,615 & 40.11 & 40.79 & 71.48 & 40.00 & 50 \\
11 & 7,384 & 15.31 & 4.86 & 66.88 & 33.33 & 40 \\
12 & 7,716 & 9.32 & 1.63 & 64.89 & 26.67 & 40 \\
13 & 7,783 & 8.40 & 1.31 & 64.49 & 20.00 & 40 \\
14 & 12,134 & 0.01 & 0.00 & 38.45 & 13.33 & 10 \\
15 & 16,901 & 0.00 & 0.00 & 9.91 & 6.67 & 0 \\
16 & 18,557 & 0.00 & 0.00 & 0.00 & 0.00 & 0 \\
\hline Mean: & $\mathbf{7 , 5 7 9}$ & $\mathbf{4 7 . 3 1}$ & $\mathbf{5 1 . 1 5}$ & $\mathbf{6 5 . 7 1}$ & $\mathbf{5 0}$ & $\mathbf{4 8 . 7 5}$ \\
\hline
\end{tabular}

Table 1: Comparison of scoring method results

10 Destatis, reference date: December 31, 2005. The per capita debt was chosen deliberately as underlying series of measurements because this indicator could be part of an actual (federal states) ranking. It was abstained from an artificially created sample in order to present a conclusive and realistic example. 
The Logistic Function Methods produce similar score values for the three best data points because they are located on the upper flat segment of the curve. Compared to this, the fourth federal state suffers a small loss of points, even if its per capita debt value is about 1,300 Euro higher. Within the interval of 6,000 and 7,000 Euro there is a larger score loss per additional debt unit. The states concerned are located on the decreasing part of the curve. Looking at countries 10 and 11 the difference in their per capita debt value merely amounts to 770 Euro. The loss of scores is reasonable high, which makes good economic sense because the remaining states exceed the critical per capita debt value of 7,000 Euro and have below-average economic results. Hardly any score differences exist among the last three states which are outliers, due to their high debt ratios. State 13 also experiences only a small score increase compared to the outliers. This ensures that results which are descending from bad to even worse, are given nearly the same score value. On the other side, economic differences between a per capita debt of 5,000 Euro (upper midfield) and 7,000 Euro (worrying) are reflected sensibly in the point allocation.

The Linear Scoring Method is compared to the Logistic Function Methods. In the underlying sample an increase of per capita debt of 167 Euro is followed by a loss of one point to maintain the scale of zero to hundred points. When applying the linear function, only the last federal state with a very high debt ratio doesn't receive scores, whereas the next two outliers have a positive score value. Data points in the midfield have higher score values which are accompanied by a lower variance compared to the Logistic Function Methods. Resulting from this compression, the altering implications of different debt ratios remain in the background. A per capita debt of more than 7,300 Euro which receives two thirds of the maximum score value can not be judged as economically plausible, when there are ten states with better results. Doubts arise if the Linear Function Method is able to produce valid results.

Using the Rank Sum Method (RSM) every country receives a score value according to its position in the data set. ${ }^{11}$ In the subjacent sample, the rank scores were transformed into the noted scale which is presented in table 2. The Rank Sum Method's weakness is that it diminishes relative distances in the data set which again makes it robust against outliers. This can be derived from the last four score values which are much smaller than they would be when using a Linear Function Method and therefore seem to be more realistic from an economical point of view. Up to the $10^{\text {th }}$ state the point allocation is similar to the scores of LF1 because the impact of the first outlier carries little weight. The masking of relative distances is at the same time strength and weakness of the Rank Sum Method. Considering federal states 11-14 shows that below-average results have received rather generous score values (up to one third of the maximum score value). Furthermore, two of the three highest outliers are provided with positive score values which take the bad debt ratio insufficiently into account.

The results of the Scoring Class Method (SCM) are comparatively good. Outliers experience consideration through the construction of adequate scoring classes. Yet again, this is the most significant disadvantage because the construction of classes requires the arbitrary determination of parameters, such as class length, transition points and score values per class. Hence, the Scoring Class Method is susceptible to criticism and will therefore suffer fewer acceptances. The summarized results are given in table 2:

\begin{tabular}{|l|c|c|c|c|c|}
\hline & Non-arbitrariness & $\begin{array}{c}\text { Elimination of } \\
\text { outliers }\end{array}$ & $\begin{array}{c}\text { Difference normal } \\
\text { / extreme values }\end{array}$ & Compression & Plausibility \\
\hline LF1 & + & + & + & + & + \\
\hline LF2 & + & + & - & + & + \\
\hline Lin. function & + & $-{ }^{*}$ & - & - & - \\
\hline RSM & + & + & + & - & + \\
\hline SCM & ${ }^{*}$ & + & & + \\
\hline Notes: & $\begin{array}{c}{ }^{*} \text { Classes need to } \\
\text { be build manually }\end{array}$ & $\begin{array}{c}{ }^{*} \text { advisable due to } \\
\text { implausible results }\end{array}$ & & & \\
\hline
\end{tabular}

Table 2: Requirements for depiction quality of scoring methods

11 Accordingly, the best result will receive 15 points, whereas the worst result gets no points. 
All methods apart from the SCM manage without random parameter determination. For LF2, parameters have to be defined but -abandoning arbitrary interventions - it is designed to make use of computer based iteration procedures. Technically, all methods can be applied without foregoing data eliminations which is at the same time the reason for implausible results of the linear function, as described above. Considerably large differences between outlier scores and the scores of the next normal values can be constituted for the linear function and RSM. The score allocation predicts that no outlier mitigation takes place. Neither the linear function nor the SCM can avoid the compression of original values which lay closely together (between 5,000 and 7,000 Euro). Weaknesses according to economically plausible results were found for the linear function and the RSM.

The previous analysis reveals that outliers might have a biasing impact on score values. At the same time, the Logistic Function Methods attracted attention in the way that they seemed to achieve highest depiction quality and valid results. The descriptive conclusion will further explore the hypothesis that logistic functions are the better choice when encountering outliers in datasets and will engage in offering proof for it.

\subsection{Validity Comparison Among Scoring Methods}

\subsubsection{Bootstrap Simulation Of Biased Samples}

Looking again at the per capita debt the sample reveals what was already assumed in the previous example, namely the existence of four outliers. Basing on the interquartile range (IQR) the first and the last three values are identified as outliers because they deviate more than 1.5 times the IQR from the $25 \%$-quantile / $75 \%$-quantile. This outlier test does not assume normal distribution and is suited for skewed samples as in the present right-skewed sample. Given the results of a Shapiro-Wilk-Test, the sample is not distributed normally. ${ }^{12}$ The same result is proven by a graphic analysis using a quantile-quantile-plot as shown in figure 2 .

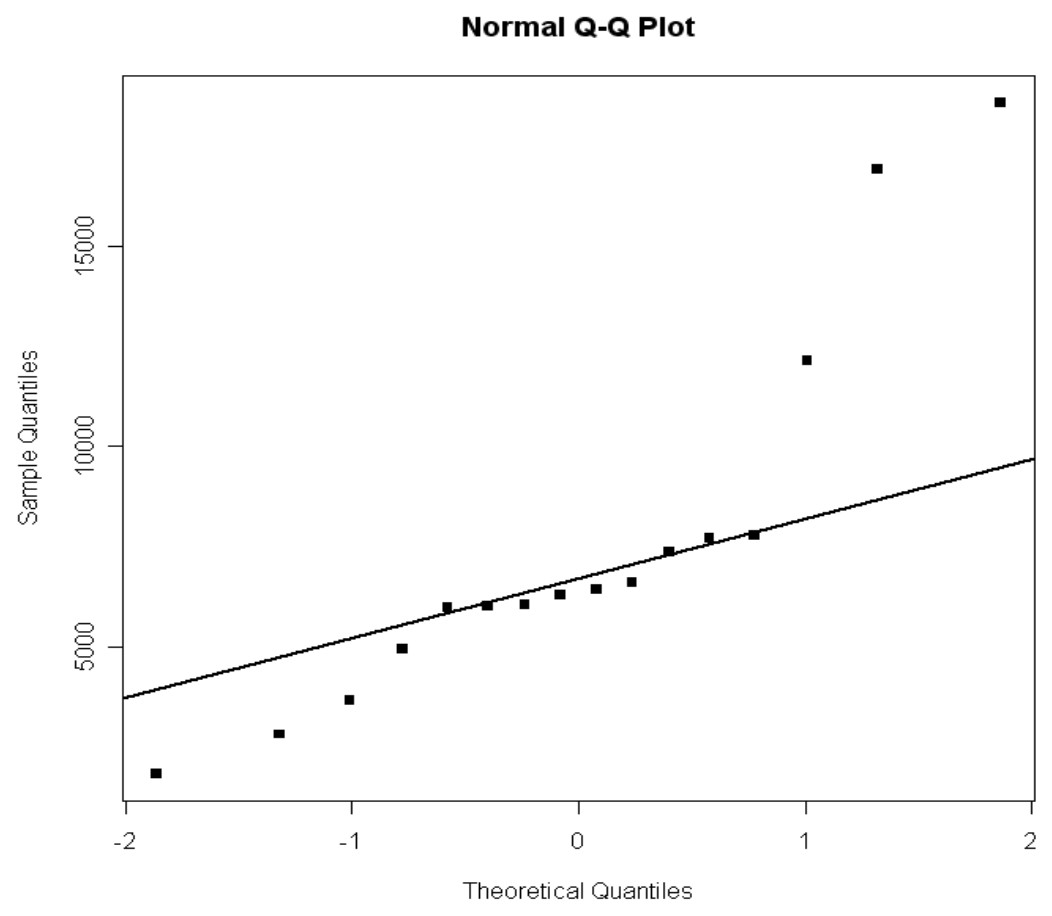

Figure 2: Normal Q-Q Plot of sample quantiles.

12 The null hypothesis is rejected with a test value of .826 and $p=.062$. 
It is disputable which scoring method is suitable for a realistic and reliable transformation of this (or similar) data sets into score values. The aggregation of a composite indicator with high validity strongly depends on the quality of the scoring technique. A comparison of scoring methods is conducted to analyze which methods grant most validity and reliability in relation to the aggregated and single indicators. Measuring the validity of a ranking order is not unimportant because the "true" ranking order (e.g. of the competitiveness of countries) cannot be observed and compared to the results of the scoring methods. By means of single indicators or economic knowledge, results can be verified or rejected at best. There is no proper procedure to test for validity so far so that a new approach has to be developed.

Jensen (2001) makes use of bootstrap rank confidence intervals to determine the "true" grading of universities and to check the validity of their position in the ranking. Following this approach, bootstrap confidence intervals on the "true" mean of a data set are computed in the next section. Each scoring method has its own strengths and weaknesses so there will probably be no prime solution for the choice of an optimal scoring method. The choice rather depends on the data structure of the single indicators of rankings. As mentioned above outliers have a strong impact on the scoring results and because of this the validity of scoring techniques is assessed in the presence of outliers.

For this purpose non-parametric bootstraps are applied on an outlier biased and a non outlier biased sample to infer the population's distribution and the mean. The question of which value will estimate the resampling mean if outliers exist and if they are omitted moves into focus. The mean was chosen as standard of comparison because it is well-known that outliers have a strong influence on it. Insofar it can be expected that the means of samples with and without outliers will be significantly different. A scoring method with the ability to mitigate outliers would be assumed to generate an average score value which (transferred back into per capita debt) comes close to the estimated non-outlier resampling mean and the surrounding confidence interval. Vice versa, the scoring mean of improper techniques will differentiate from the resampling mean without outliers. The statistical feature of the s-shaped logistic curve might lead to a decreasing mean in the case of high extreme values and an increasing mean in the case of small extreme values.

Developing bootstrap percentile confidence intervals around the demanded statistic will help to analyze the unknown distribution of the "true" mean in an outlier biased sample. The central assertion of this approach is that the relative frequency distribution of the estimated resampling means $\hat{\theta}^{*}$ is the best approximation of the sampling distribution of $\hat{\theta}$. The sampling distribution is used to develop intervals around $\hat{\theta}$ in which $\theta$ (the true sample mean) can be found with a known probability. ${ }^{13}$ Having estimated the sample distribution, inferences about the mean's location can be deviated given a probability of $95 \%$.

Conclusions about the parameter which are done with classic inference methods are likely to be biased because the sample is not normally distributed. Additionally, a normal distribution cannot be assumed employing the central limit theorem because the sample size of $n=16$ observations is too small. As a result, it is not reasonable to apply classic inference methods whereas for the same reasons the application of the bootstrap method makes good sense.

In a first step the mean $\hat{\theta}$ is calculated from the original sample:

$$
\bar{x}^{o}=\frac{1}{2} \Sigma\left(x_{i}\right)^{14}
$$

13 The resamples of size $\mathrm{n}=1,000$ are drawn from the sample randomly with replacement. Therefore, the resamples will differ randomly from the sample values. As a function of $\hat{\theta}, \hat{\theta}^{*}$ is distributed randomly in the same way as $\hat{\theta}$ (Duval / Mooney: 21).

14 Index „o“ means with “outliers” and index „no“ means “no outliers”. 
In case of per capita debt and in the presence of (large positive) outliers the mean amounts to 7,579, whereas the median is 6,379.5. It becomes apparent that the mean is susceptible to outliers. In the next step four outliers ${ }^{15}$ are eliminated and the new mean is calculated from the adjusted sample: $\bar{x}^{n o}=5,985$. The next figure shows density, median (solid line) and mean (dashed line) of both samples employed. Observing the adjusted sample it is clearly recognizable that the new mean has decreased and lies closer to the sample median. This can be explained by the elimination of three large right-sided extreme values.
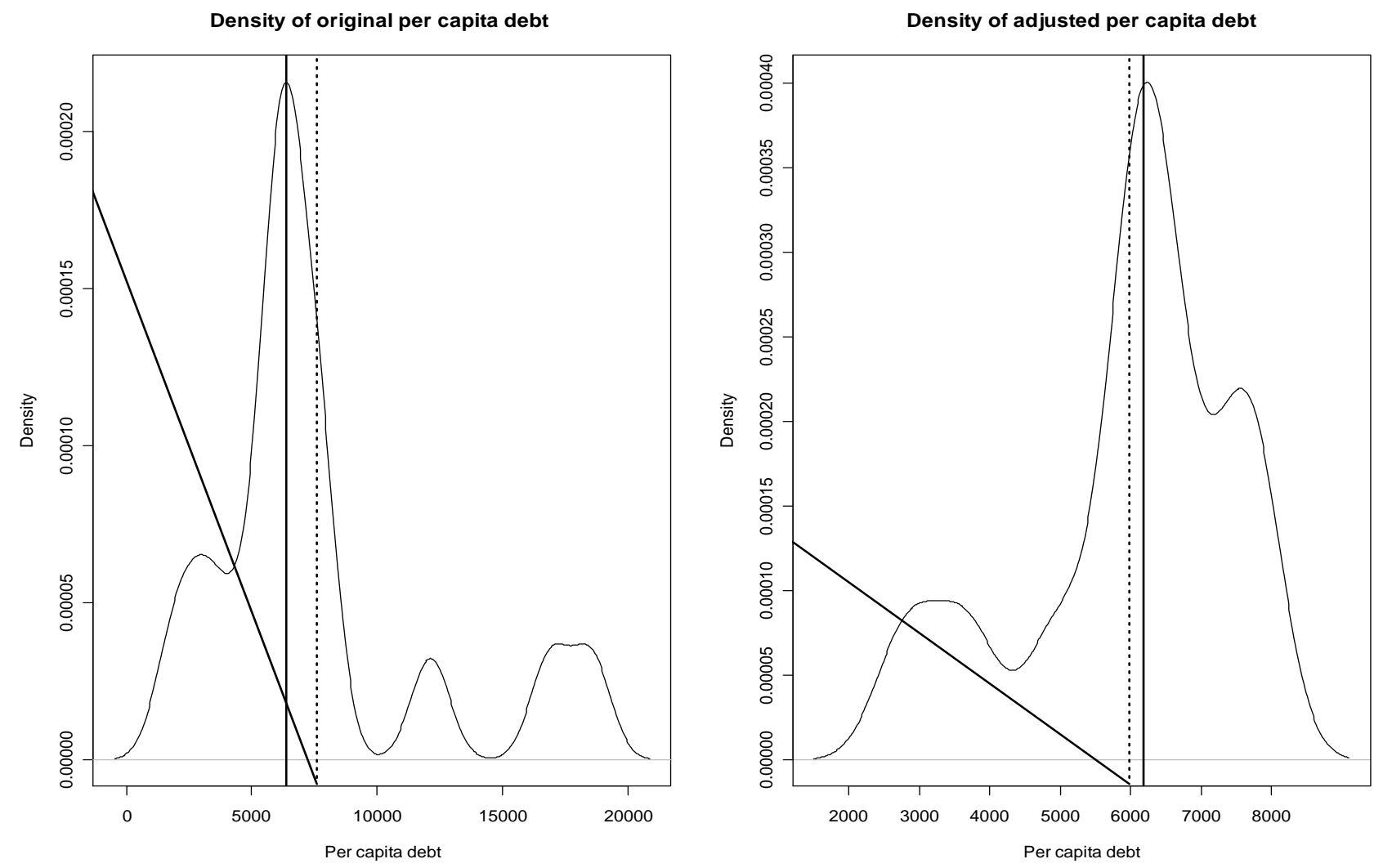

Figure 3: Density, median and mean of the chosen samples.

For the estimation $\mathrm{B}=1,000$ resamples were drawn from the samples with and without outliers. The size of 1,000 resamples was recommended by Mooney and Duval (1993 and 1996) and in the fundamental works of Efron and Tibshirani (1986 and 1993) for the application of bias-corrected confidence intervals (BC method). The absolute frequency distribution of the resampling means is shown in the following figure.

While the resampling means, drawn from the outlier biased sample centre upon an interval from 7,000 to 8,000 Euro, the highest absolute frequency of means without outliers ranges from 5,750 and 6,250 Euros.

$15 \quad(1,851 ; 12,134 ; 16,901 ; 18,557)$ 
Resampling means

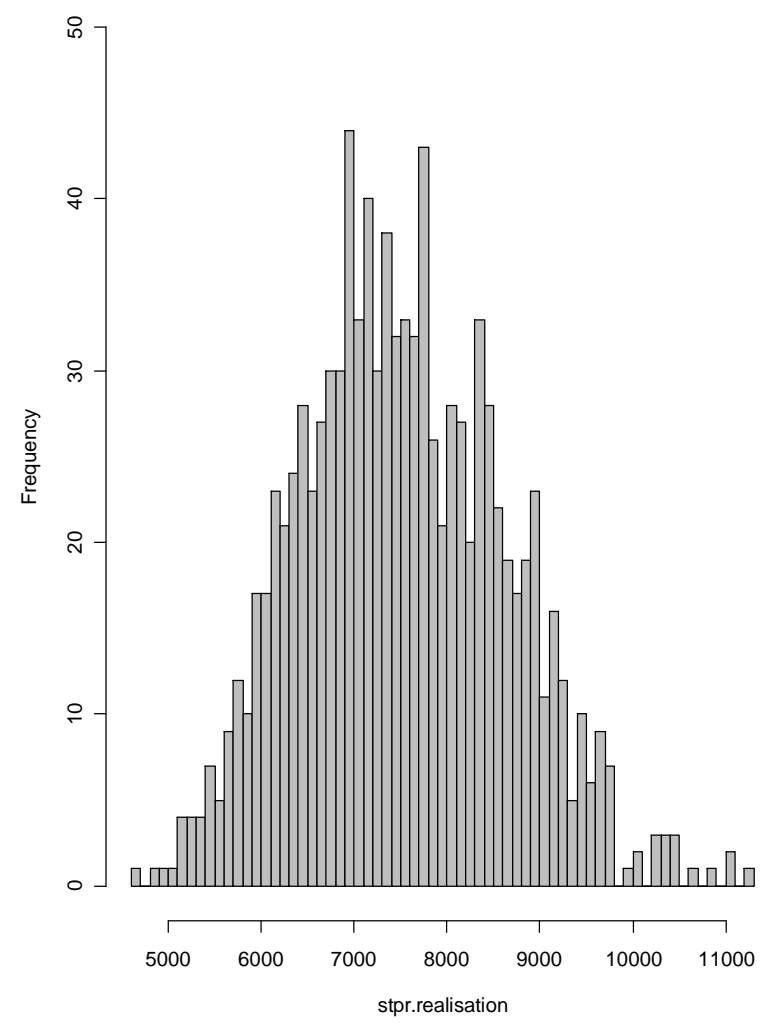

Resampling means of the adjusted sample

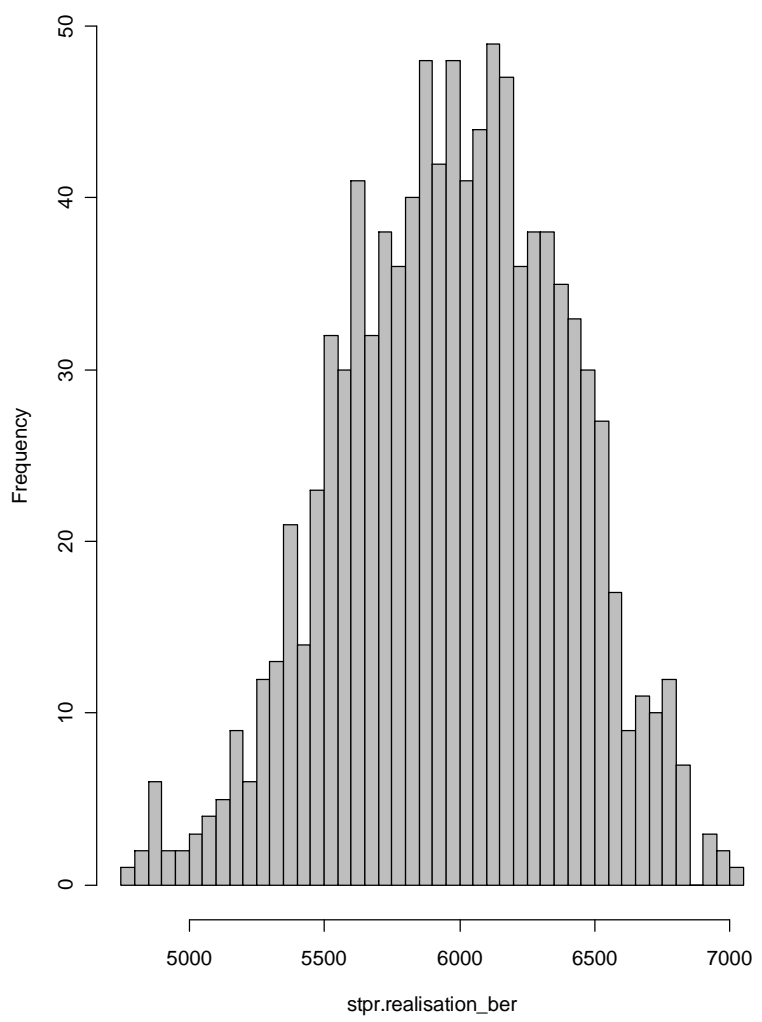

Figure 4: Absolute frequency distribution of resampling means.

\subsubsection{Validity Comparison By Means Of Bootstrap Confidence Intervals}

Next to the resampling process, confidence intervals are developed around the estimated means. The confidence intervals admit probability statements about the location of the demanded statistic and offer more information than a point estimation of the mean. There are several techniques using the bootstrap sampling distribution to build confidence intervals around the population parameter. The following methods are most common:

- $\quad$ normal approximation method

- $\quad$ percentile method

- $\quad$ bias-corrected method and

- $\quad$ percentile-t method.

The choice of a technique depends on bias, skewness and kurtosis of the resamples. When it is plausible to assume that the statistic is normally distributed then the normal approximation method can be used (Mooney / Duval 1993: 35). The bootstrapped sample mean was tested on a probable normal distribution with a Shapiro-Wilk-Test which is not able to verify the assumption. The optical comparison of density functions of resampling means and normal distribution backs up the test result. 

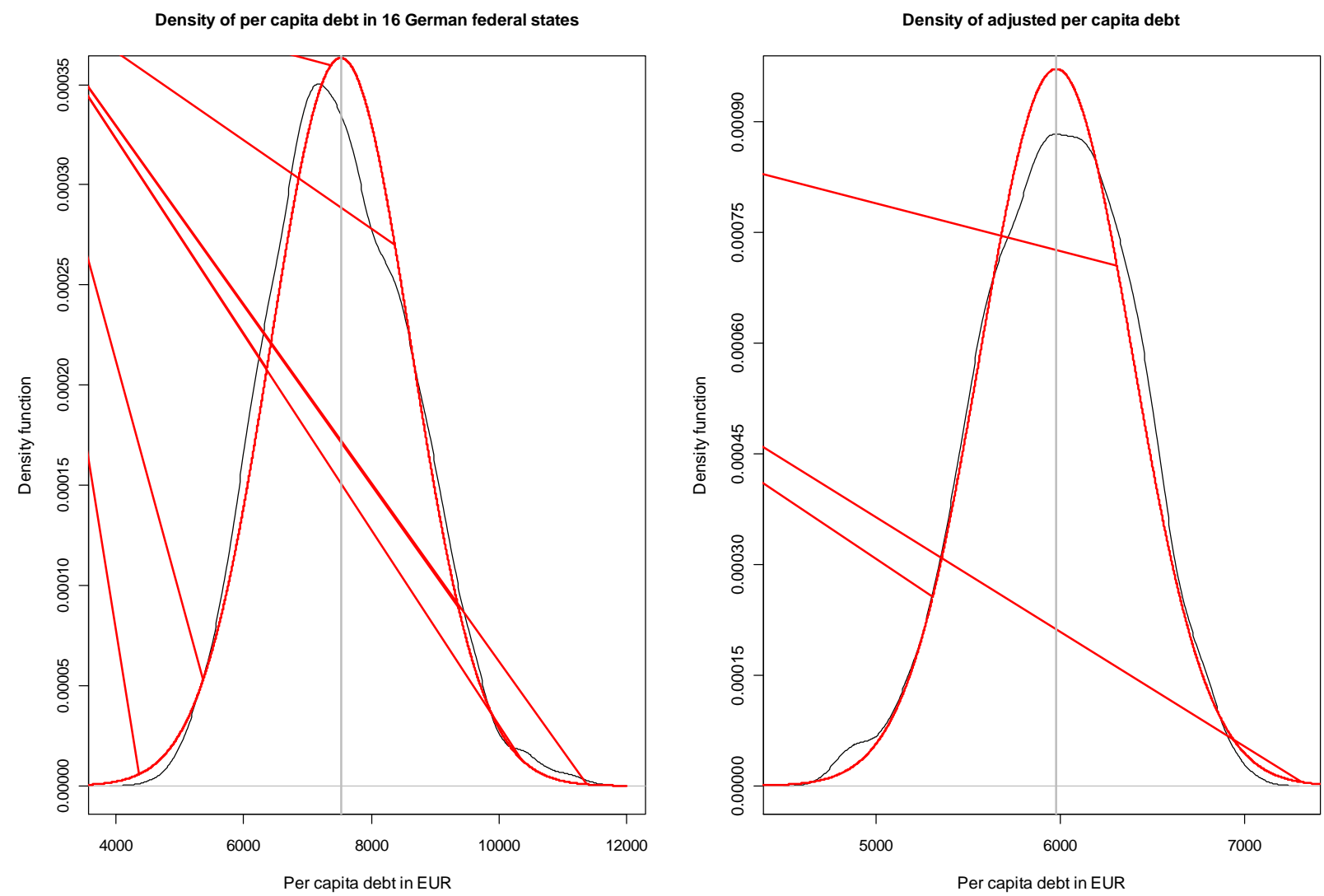

Figure 5: Density of Resampling means compared to the normal distribution density.

The application of the bias-corrected or BC method is advisable if there is a bias between the estimated resampling mean $\hat{\theta}^{*}$ and the sample mean $\hat{\theta}$. A bias of zero would speak for the percentile method (i.e. in the case of the per capita debt sample). Following Mooney and Duval (1993:33) the BC method should be applied if the ratio of the estimated bias to the standard error is not less than .25 . The bias was estimated by: ${ }^{16}$

$$
\operatorname{Estimate}[\operatorname{Bias}(\hat{\theta})]=\hat{\theta}-\hat{\theta}_{(.)}^{*}, \quad \text { where } \hat{\theta}_{(.)}^{*}=\Sigma \hat{\theta}^{*} / B
$$

It amounts to 60.0 (with outliers) and 7.18 (adjusted sample). The estimated standard error $\hat{\sigma}^{*}$ is given by:

$$
\hat{\sigma}^{*}=\sqrt{\frac{\sum\left[\hat{\theta}^{*}-\left(\hat{\theta}^{*} / B\right)\right]^{2}}{(B-1)}} .
$$

Referred to the resampling results the values are 1,098.3 (with outliers) and 411.0 (adjusted sample). The ratios between bias and standard error therefore amount to .055 and .017 and fall below the critical value.

16 The estimated bias is the difference between the sample mean and the expected value of the bootstrapped sampling distribution. 
Skewed distributions favorably suggest the application of the percentile-t-method. The per capita debt resamples were checked for skewness which was found to be negligible (.037 and -.024).

Briefly summarized, the percentile method can be used to calculate confidence intervals. This method has the advantages of allowing for an asymmetrical sample distribution $F(\hat{\theta})$ and an easy handling. Moreover the percentile method does not require parametric assumptions about the sample distribution (e.g. normal distribution). Additional information is given by $\mathrm{BC}$ confidence intervals which were calculated to compensate the minor bias. The reason is that the percentile method assumes the resampling distribution $\hat{F}^{*}\left(\hat{\theta}^{*}\right)$ to be unbiased.

Regarding the outlier biased sample the percentile method yields a confidence interval for the mean of:

$$
\mathrm{p}(5.554<\mu<9.673)=.95
$$

The average per capita debt is 7,579 Euro. Using the BC method the interval endpoints were found at the $98.46^{\text {th }}$ and $3.92^{\text {th }}$ percentile of $\hat{F}^{*}\left(\hat{\theta}^{*}\right)$ and the result changes to:

$$
\mathrm{p}(5.729<\mu<10.012)=.95 .
$$

Taking the small bias into account a right-sided shift of the interval endpoints can be constituted. The upper endpoint diverges more than the lower endpoint which is the result of the asymmetry correction between the resampling and sampling distribution. The unequal relocation of interval endpoints enables the centering of the bootstrapped sampling distribution $\hat{F}^{*}\left(\hat{\theta}^{*}\right)$ on the point estimator, $\hat{\theta}$ (Mooney / Duval 1993: 39).

The percentile interval endpoints of the bootstrapped sample without outliers result in:

$$
\mathrm{p}(5.153<\mu<6.745)=.95
$$

The average resampling mean (namely $\Sigma \hat{\theta}^{*} / B$ ) is equal to 5,978 Euro. The endpoints of the BC method are 5,157 and 6,754 Euro. ${ }^{17}$ The interval shows only a sparse displacement and merely compensates for a minor rest asymmetry. Both confidence intervals have become narrower than those in the outlier case and the endpoint values have decreased. The reduced interval range improves drawing conclusions about the "true" per capita debt values if outliers have no influence. While the interval range in the outlier sample is about 4,119 Euro, the range has decreased to 1,592 Euro in the case of the adjusted sample which limits the range value in which the demanded statistic can probably be found.

In the next step, the percentile interval of the sample without outliers is used as a standard of comparison for the ability of various scoring methods to mitigate outliers. The data set of Germany's federal states' per capita debt is transformed into scores. The average score value is calculated and recalculated into a value of per capita debt. If the applied scoring method has the ability to mitigate outliers, the determined value should come close to the estimated mean and fall into the confidence interval. The results are presented in table 3.

17 The endpoints were found at the $97.62^{\text {th }}$ and $2.62^{\text {th }}$ percentile. 


\begin{tabular}{|lcc|}
\hline & Average score value & Per capita debt \\
\hline LF1 & & 6,511 \\
LF2 & 47.25 & 6,491 \\
Lin. function & 51.15 & 7,579 \\
RSM & 65.71 & 6,380 \\
SCM & 50.00 & $7,001-8,000$ \\
Est. resampling per capita debt & 48.75 & \\
Bootstrap confidence interval & & $\mathbf{5 , 9 7 8}$ \\
\hline
\end{tabular}

Table 3: Comparison of average point scores and bootstrap confidence interval

The score values of the Logistic Function Methods imply a mean per capita debt which exceeds the point estimator of the mean without outliers but falls into the developed percentile confidence interval. The results of both functions come close to the estimated mean without a need for previous data elimination or arbitrary interventions. This supports the hypothesis that Logistic Function Methods have special characteristics for the mitigation of outliers. At the same time it becomes obvious that the logistic techniques fail to eliminate the outlier effects completely. The average score value of the linear function is equivalent to a per capita debt of 7,579 Euro which is exactly the same value as the mean of the original sample with outliers. This result is not surprising, because nothing else may be expected from a linear score transformation. As a result, the biasing outlier effect is still unmitigated. Likewise the per capita debt value is far away from the estimated resampling mean and the confidence interval. The corresponding debt ratio of the SCM is larger than the estimated mean and does not fall into the confidence interval which can be attributed to the constructed classes. Using adequate classes can improve this result but only at the expense of higher arbitrariness. Additionally, the definition of classes requires a great effort, especially in the multi-indicator case.

Surprisingly, the calculated average per capita debt of the RSM comes closer to the estimated mean than any other value. As described above, the RSM shows robustness against outliers, because relative distances are ignored. This implies that the large distance between outliers and next normal values carries little weight and therefore has no overproportional impact on the point allocation. Compared to the linear function, outliers gain smaller score values and have smaller score differences compared to the remaining values. The robustness is the reason for the average per capita debt coming closer to the estimated mean. At the same time it must be considered that the calculated per capita debt corresponds to the median of the data set, which is naturally unaffected by outliers. At this point a weakness of the formal approach becomes apparent as only one parameter is used as standard of comparison for the validity of a scoring method. To bring forth a comprehensive evaluation it is necessary to combine the results of the formal and the theoretical approach shown in table 3. According to this augmented analysis, the evaluation of the RSM is in need of further differentiation. It becomes apparent that masking relative distances on the one hand leads to some robustness against outliers and on the other hand accounts for unrealistic depiction of data points and implausible score values.

\section{CONCLUSION}

Applying logistic functions as a scoring method is recommendable if a data set consists of "normal" data points which lie closely together and show few extreme values. The biasing impact of outliers is compensated in large samples but when small samples are used outliers tend to compress score values of normal data points and cause invalid point allocations. Many rankings make use of single, small sized indicators. Such samples with less than 20 data points are vulnerable to outliers and need the application of an adequate scoring method which possesses the ability to mitigate outlier distortion effects. Under these conditions Logistic Function Methods are robust techniques which allow for valid and realistic point allocations as established within the combined formal and theoretical analyses. To a certain degree the Rank Sum Method and Scoring Class Method produce valid results if arbitrariness is countenanced, thus giving way for (further) criticism. 
If the data set is large or outliers do not exist, the Linear Function Method will serve well. If an extreme value is a "true" outlier, which means that it is not the result of specific economic efforts or it is a random singular occurrence and insofar doesn't serve as a benchmark the Logistic Scoring Method should be applied.

However, it should be thoroughly considered that before changing the methodology of a ranking it is advisable to assess if the chosen technique can be maintained. The reason is that the advantage of a transparent and consistent methodology can overcompensate for unfavorable "automatic" outlier mitigation. In the case of a preferred "benchmark-outlier" the problem can be solved by the determination of eligible parameters (for example a small value for $\mathrm{c}$ in equation three) which gives a higher weight to extreme values. The definition of parameters then depends on the ranker's conviction, historical comparisons and other preliminary tests if the maximum and minimum values measured are "true" outliers which need to be smoothed or if they make good economic sense.

Finally, outlier effects can not be eliminated completely. They can only be mitigated to some extend which was shown by the use of bootstrap percentile confidence intervals. Furthermore, the elimination of the extreme values themselves is no appropriate method to control for outliers because it would mean an arbitrary intervention which is a target for criticism. Acceptance of the methodology is of vital importance for the success of rankings. Therefore the elimination of data points should be avoided. Using the logistic scoring method can help to improve validity and reliability of modern rankings.

\section{AUTHOR INFORMATION}

\section{Nicole Uhde}

Doctorand in Economics

Born: 4th. March 1980, Birthplace: Hamm (North Rhine-Westphalia, Germany). After an apprenticeship in the Dresdner Bank AG she has studied economics in Muenster and finished in 2006 with a Master's degree (Master of Science in Economics). Since January of 2007 she is working as research assistant at the Institute of Spatial and Housing Economics of the University of Muenster. Besides, she is teaching at the University of Applied Sciences in public administration in Muenster.

\section{REFERENCES}

1. Arrow, K. (1963): Social Choice and Individual Values. 2. Ed. New Haven.

2. Barnett, Vic / Lewis, Toby (1978): Outliers in Statistical Data. Chichester a.o.

3. Barnett, Vic / Lewis, Toby (1994): Outliers in Statistical Data. 3. revised Ed. Chichester.

4. Bayer, Christian R. (2000): Eine Analyse des entscheidungsunterstützenden Informationsgehaltes deutscher und US-amerikanischer Hochschul-Rankings. Discussion Paper Nr. 593, Universität Karlsruhe (TH). Karlsruhe.

5. Kane, Tim et al. (2007): 2007 Index of Economic Freedom. Washington.

6. Belsley, David A. et Al. (1980): Regression Diagnostics: Identifying Influential Data and Sources of Collinearity. New York a.o.

7. Buttler, Günter (1996): Ein einfaches Verfahren zur Identifikation von Ausreißern bei multivariaten Daten. Diskussionspapier Nr. 9 der Wirtschafts- und Sozialwissenschaftlichen Fakultät der Universität ErlangenNürnberg.

8. Bertelsmann Stiftung (2007): Internationales Standort-Ranking 2007. Gütersloh.

9. Efron, Bradley / Tibshirani, R. (1986): Bootstrap methods for standard errors, confidence intervals, and other measures of statistical accuracy. In: Statistical science, 1(1), 54-75. Hayward.

10. Efron, Bradley / Tibshirani, Robert J. (1993): An introduction to the bootstrap. New York a.o.

11. Giovannini, Enrico et al. (2005): Handbook on constructing Composite Indicators: Methodology and User Guide. In: OECD Statistics Working Papers, No. 3.

12. Grubbs, Frank E. (1969): Procedures for detecting outlying observations. In: Technometrics, 11, 1-21. Rochester. 
13. Jensen, Uwe (2001): Bootstrap-Rangkonfidenzintervalle für Hochschul-Ranglisten. In: Detlef MüllerBöling et al. (Hrsg.): Tagung Hochschulranking. Aussagefähigkeit, Methoden, Probleme. (pp. 65-89). Gütersloh.

14. Kladroba, Andreas (2005): Statistische Methoden zur Erstellung und Interpretation von Rankings und Ratings. 1. Ed. Berlin.

15. Kladroba, Andreas (2005 a): Methodische Einflüsse auf die Ergebnisse von Rankings. Einige Anmerkungen am Beispiel des Bertelsmann Standort-Rankings 2004. In: Dieter Duwendag et al. (Hrsg.): Jahrbuch für Wirtschaftswissenschaften, 56(1), 95-111. Göttingen.

16. Martin, David / Kumar, Vikram (2005): Detecting outliers and influential observations with heteroscedasticity-corrected models. In: Applied economics letters, 12(12), 745-748. Abingdon.

17. Matthes, Jürgen / Schröder, Christoph (2004): Rahmenbedingungen für Unternehmen - zur Aggregation von Weltbankdaten. In: Institut der Deutschen Wirtschaft (Hrsg.): IW-Trends. Vierteljahresschrift zur empirischen Wirtschaftsforschung aus dem Institut der deutschen Wirtschaft Köln, 31(4), 51-62. Köln.

18. Mooney, Christopher Z. / Duval, Robert D. (1993): Bootstrapping. A Nonparametric Approach to Statistical Inference. Sage University Papers Series No. 07-095. Newbury Park a.o.

19. Mooney, Christopher Z. / Duval, Robert D. (1996): Bootstrap Statistical Inference: Examples and Evaluations for Political Science. In: American Journal of political science, 40, 570-602. Malden.

20. Ochel, Wolfgang / Röhn, Oliver (2006): Ranking of Countries - The WEF, IMD, Fraser and Heritage Indices. In: CESifo Dice Report, 2, 48-60. München.

21. Statistisches Bundesamt (2007): Statistisches Jahrbuch 2007. Wiesbaden.

22. Sturm, Jan-Egbert / de Haan, Jakob (2000): No need to run millions of Regressions. CESifo Working Paper Series No. 288. München.

23. Van Suntum, Ulrich (2004): Methodische Probleme des Benchmarking - Der Bertelsmann-Bench-markingIndex. In: Beiträge zur angewandten Wirtschaftsforschung Nr. 3. Münster.

24. World Economic Forum (2004): The Global Competitiveness Report 2003-2004. New York u.a. 


\section{NOTES}

